\title{
Fibrin Monomer
}

National Cancer Institute

\section{Source}

National Cancer Institute. Fibrin Monomer. NCI Thesaurus. Code C107563.

Derivatives of thrombin-cleaved fibrinogen that are plasma soluble due to limiting amounts of the clot promoting protein factor XIII. Detection of these proteins may be used to aid in the diagnosis or exclusion of thrombotic events or determine if a patient is at risk for post-operative hemorrhage. 